\title{
A Comparative Study of Multi-Objective Evolutionary Trace Transform Methods for Robust Feature Extraction ${ }^{\star}$
}

\author{
Wissam A. Albukhanajer ${ }^{1}$, Yaochu Jin ${ }^{1}$, Johann A. Briffa ${ }^{1}$, and \\ Godfried Williams ${ }^{2}$ \\ 1 University of Surrey, Faculty of Engineering \& Physical Sciences, \\ Department of Computing, \\ Guildford, Surrey, GU2 7XH, United Kingdom

\begin{abstract}
Recently, Evolutionary Trace Transform (ETT) has been developed to extract efficient features (called triple features) for invariant image identification using multi-objective evolutionary algorithms. This paper compares two methods of Evolutionary Trace Transform (method I and II) evolved through similar objectives by minimizing the withinclass variance $\left(S_{w}\right)$ and maximizing the between-class variance $\left(S_{b}\right)$ of image features. However, each solution on the Pareto front of method I represents one triple features (i.e. 1D) to be combined with another solution to construct 2D feature space, whereas each solution on the Pareto front of method II represents a complete pair of triple features (i.e. 2D). Experimental results show that both methods are able to produce stable and consistent features. Moreover, method II has denser solutions distributed in the convex region of the Pareto front than in method I. Nevertheless, method II takes longer time to evolve than method I. Although the Trace transforms are evolved offline on one set of low resolution $(64 \times 64)$ images, they can be applied to extract features from various standard $256 \times 256$ images.
\end{abstract}

Keywords: Evolutionary algorithms, multi-objective optimization, Pareto optimality, Trace transform, image identification, invariant feature extraction.

\section{Introduction}

Identification of digital images is challenging as pictures of the same object will look very different taken from different angles, distances and lighting conditions.

\footnotetext{
* This work is supported by EPSRC and Intellas UK Ltd.
} 
Further, images acquired by cheap consumer cameras are usually noisy and differ by different camera specifications [1]. Therefore, a robust image identification requires extracting image features independent of the way the objects are presented in the image.

Correspondingly, extracted features should be insensitive to variations in geometric transformations such as rotation, scale and translation (RST). Additionally, features derived from different samples of the same image class should be similar. Conversely, features derived from samples of different image classes (see Fig. 1) should considerably differ from each other.

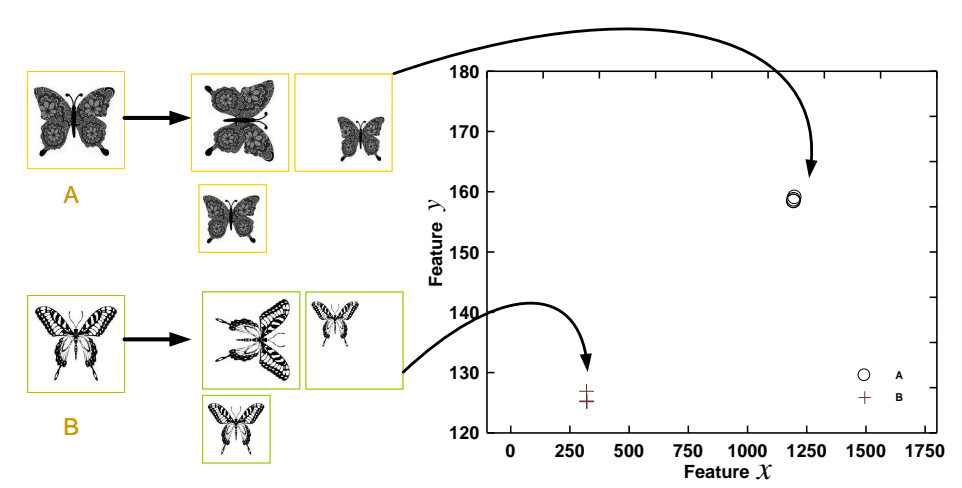

Fig. 1: Mapping of two image classes into a 2D invariant feature space.

Trace transform (TT) [2] calculates functionals on image pixels along straight lines projected in different orientations. For example, Fig. 2 depicts an image and its Trace transform produced by using the first Trace functional in Table 1 (the integral of a function). Refer to Fig. 2, the transform matrix in Fig. 2c is obtained by tracing the image (Fig. 2b) with straight lines characterized by a length $\rho$ and an angle $\theta$, and calculating a functional called "Trace" $T$ over parameter $t$ (see Fig. 2a). Therefore, the transform matrix is a 2D image of $\rho \times \theta$ pixels (Fig. 2c). Different transforms can be obtained by using different Trace functionals.

With the help of a second functional called "Diametric" $D$ evaluated along columns of Trace matrix (i.e. along parameter $\rho$ ), a string of numbers is created

Table 1: List of some Trace functional

\begin{tabular}{ccc}
\hline \hline No. & Functional & Description \\
\hline 1 & $\int f(t) d t$ & Radon transform \\
2 & $\int\left|f(t)^{\prime}\right| d t$ & Integral of Gradient \\
3 & $\left.\left.\left(\int \mid f(t)\right)\right|^{p} d t\right)^{q}$ & p-Norm, $p=0.5, q=1 / p$ \\
4 & $\max -\min (|f(x)|)$ & Maximum-minimum of the function \\
\hline \hline
\end{tabular}




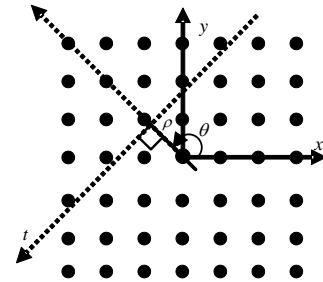

(a) The Trace parameters [2]

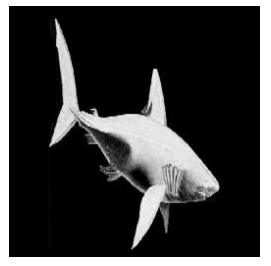

(b) Fish image.

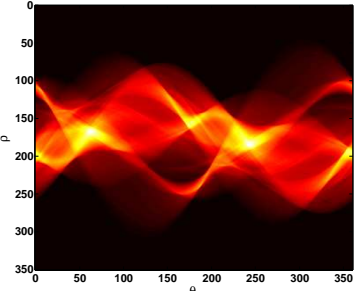

(c) Its Trace Transform.

Fig. 2: The Trace Transform

with a length of $\theta$. Finally, a third functional called "Circus" $C$ is applied to the final string of numbers (over parameter $\theta$ ) to produce a scalar value "real number". This number is termed a "Triple" feature denoted by $\prod$ and it can be used to form a unique identifier for the image [3].

To characterize an image, features derived by Trace transform are not necessarily transparent to the human perception such as brightness or color. In fact, thousand of features can be constructed using different Trace transform functionals by using different combinations of these functionals. Therefore, one may consider Trace transform as a mathematical tool to represent brain sub-conscious which we can not usually identify [4].

Trace Transform has been successfully applied to many image processing tasks such as image database retrieval [2], texture classification [4] and characters recognition [5]. Evolutionary Trace Transform (ETT) [6] has recently been developed to construct efficient Trace transform triple features to represent an image. The main idea is to find optimal combinations of the functionals together with the number of projections in Trace transform to achieve fast and robust feature extraction. It has been shown that evolutionary Trace transform is more robust and efficient than the traditional Trace transform [6].

This paper compares two methods of Evolutionary Trace Transform, method I and II, developed using multi-objective evolutionary optimization of Trace transform to produce candidate features of digital images. The remainder of the paper is organized as follows. Section 2 presents a brief overview of the ETT. Both methods are given as two different evolutionary methods to evolve the Trace transform. Section 3 depicts the experimental results for performance evaluations. Finally, a conclusion is given in Section 4.

\section{Evolutionary Trace Transform}

A variety of Trace functionals can be employed in Trace transform to extract features that may represent an image. However, robustness and computational 
speed are two important factors for efficient image analysis. Therefore, it is crucial to design an algorithm to construct efficient features.

An attempt to use evolutionary algorithms in Trace transform was reported by Liu and Wang [7] for face recognition. The authors have introduced a hybrid Trace features from multiple rotation-based Trace functionals and a traditional Genetic Algorithm (GA) to optimize a scalar variable associated with each trace feature. In [8] a reinforcement learning algorithm was applied to the weighted Trace transform (WTT) to find the optimal threshold in the WTT space to minimize the within-class variance only. Recently, in [6], an Evolutionary Trace Transform (ETT) is developed for invariant feature extraction. It has been shown that ETT outperforms the traditional TT in extracting robust triple features from images.

ETT employs a Pareto optimization method, the NSGA-II to search for optimal functional combinations that trade off between minimizing within-class variance and maximizing between-class variance of triple features represent an image. However, the extracted features are one-dimensional. If two-dimensional features are to be extracted from images, then two pairs of functionals need to be constructed. An alternative is to optimize Trace functionals by directly extracting two-dimensional features. Therefore, in the following, we will discuss these two approaches in greater details.

\subsection{Method I}

In this method, each solution in the Pareto-front represents a single triple feature. Then, two solutions from the final Pareto-front are randomly selected (denoted by $\prod_{x}^{(I)}$ and $\prod_{y}^{(I)}$ ) to form a $2 \mathrm{D}$ feature space. In the following, the main components of method I are presented.

- Chromosome: Each chromosome in method I encodes 4 integer parameters for each triple feature, namely, Trace $T 1$, Diametric $D 1$, Circus $C 1$ and $\theta 1$.

- Population: The population size is initialized randomly with constraints on the design variables. For example, there are 14 Trace functionals, then $T 1$ change from 0 to 13 .

- Fitness: The fitness function is characterized by two objectives which are set for minimization in the evolutionary algorithm. The two objectives are defined in (1):

$$
\begin{aligned}
& f_{1}=S_{w}^{I} \\
& f_{2}=1 /\left(S_{b}^{I}+\epsilon\right)
\end{aligned}
$$

where $\epsilon$ is a small quantity to avoid division by zero. $S_{w}^{I}$ and $S_{b}^{I}$ are the within-class variance and between-class variance defined in (2): 


$$
\begin{aligned}
& S_{w}^{I}=\sum_{k=1}^{K} \sum_{j=1}^{N_{k}}\left(x_{j k}-\mu_{k}^{x}\right)^{2} \\
& S_{b}^{I}=\sum_{k=1}^{K}\left(\mu_{k}^{x}-\mu^{x}\right)^{2}
\end{aligned}
$$

where

$$
\mu_{k}^{x}=\frac{1}{N_{k}} \sum_{j=1}^{N_{k}} x_{j k}, \mu^{x}=\frac{1}{K} \sum_{k=1}^{K} \mu_{k}^{x}
$$

and $K$ : number of classes, $N_{k}$ : number of samples in class $k, \mu_{k}^{x}$ : mean of class $k$ of $x$ triple features, $x_{j k}$ : the $j^{\text {th }}$ sample of class $k$ of $x$ triple features, and $\mu^{x}$ : mean of all classes of $x$ triple features.

\section{$2.2 \quad$ Method II}

Pareto-optimal solutions in method II are a set of optimal triple features that describe an image by extracting a pair of triple features (denoted by $\prod_{x}^{(I I)}$ and $\prod_{y}^{(I I)}$ ) instead of a single triple feature. That is, each Pareto-optimal solution represents a complete $2 \mathrm{D}$ feature space. In the following, the main components of method II are presented.

- Chromosome: Each chromosome in method II encodes 8 integer parameters instead of 4 only in method I, namely, trace $T 1, T 2$, diametric $D 1, D 2$, circus $C 1, C 2$ and $\theta 1, \theta 2$ for each triple feature.

- Population: The population size is fixed and similar to method I and initialized randomly with constraints on all design variables. For example, there are 14 Trace functionals, then $T 1$ and $T 2$ change from 0 to 13.

- Fitness: The fitness function in (3) is similar to method I where $f_{1}$ and $f_{2}$ are minimized in the evolutionary algorithm. However, the two objectives $S_{w}^{I I}$ and $S_{b}^{I I}$ are determined based on a pair of triple features defined in (4).

$$
\begin{aligned}
& f_{1}=S_{w}^{I I} \\
& f_{2}=1 /\left(S_{b}^{I I}+\epsilon\right)
\end{aligned}
$$

where $\epsilon$ is same as in (1).

$$
\begin{aligned}
& S_{w}^{I I}=\sum_{k=1}^{K} \sum_{j=1}^{N_{k}}\left(x_{j k}-\mu_{k}^{x}\right)^{2}+\left(y_{j k}-\mu_{k}^{y}\right)^{2} \\
& S_{b}^{I I}=\sum_{k=1}^{K}\left(\mu_{k}^{x}-\mu^{x}\right)^{2}+\left(\mu_{k}^{y}-\mu^{y}\right)^{2}
\end{aligned}
$$


where

$$
\mu_{k}^{x}=\frac{1}{N_{k}} \sum_{j=1}^{N_{k}} x_{j k}, \mu_{k}^{y}=\frac{1}{N_{k}} \sum_{j=1}^{N_{k}} y_{j k}, \mu^{x}=\frac{1}{K} \sum_{k=1}^{K} \mu_{k}^{x}, \mu^{y}=\frac{1}{K} \sum_{k=1}^{K} \mu_{k}^{y}
$$

and $K$ : number of classes, $N_{k}$ : number of samples in class $k, \mu_{k}^{x}$ : mean of class $k$ of $x$ triple features, $\mu_{k}^{y}$ : mean of class $k$ of $y$ triple features, $x_{j k}$ : the $j^{\text {th }}$ sample of class $k$ of $x$ triple features, $y_{j k}$ : the $j^{\text {th }}$ sample of class $k$ of $y$ triple features, $\mu^{x}$ : mean of all classes of $x$ triple features and $\mu^{y}$ : mean of all classes of $y$ triple features.

The following operations are similar for both methods:

- Selection: Selection operation is performed twice in the evolutionary loop. The first selection is performed to select parents for mating. It has been shown [9] that the tournament selection has better or equivalent convergence and computational time complexity compared to any other selection operators that available in the literature and, therefore, it is adopted in this work. In a tournament selection, two solutions are chosen from the population and a tournament is played, a good solution wins and placed in the mating pool. This operation repeats and two other solutions are played. The better solution wins and placed to fill the mating pool. Each solution will participate twice in the tournament and better solutions will win twice, and therefore two copies may exist in the population to replace the bad solutions. The second selection occur after mating to produce new population for the next generation. The elitist NSGA-II based selection [9] is adopted which consists of four steps. First, parents and offsprings are merged in one population. Second, a non-dominated sorting (Pareto-front assignment) is performed. Each non-dominated solution is assigned a Pareto-front rank number 1 (first non-dominated front), then the next non-dominated solutions in the population are identified and assigned Pareto-front rank number 2 (second non-dominated front). By repeating this procedure, a set of $r$ Pareto-fronts are generated. The third step involves sorting all solutions in an ascending order according to the assigned Pareto-front rank number, the solutions that have the same Pareto rank number are sorted in an decreasing order according the the crowding distance, and a solution with larger (better) crowding distance survives. The reader is referred to [9] for details about Crowding Distance calculation. Finally, the top individuals that fit the population size are selected and passed to the next generation.

The selection operation is performed on the combined population to generate new parents for the next generation. This preserves the good parents to survive to the next generations.

- Recombination: Two parents are selected for mating by exchanging (crossing over) a portion of information between parents in the mating pool. The crossover performed in variable ways depending on the position of the allels 
to be exchanged, i.e. at single point (one-point crossover), two points (twopoints crossover) or at an allel level (uniform crossover). In this work we adopt uniform crossover. Crossover operation occurs during the evolution at crossover probability $P_{c}$.

- Mutation: The next operation during evolution is Mutation operation which is performed to prevent the population from falling into a local optimum. The mutation is performed by inverting the gene value in an individual at mutation probability $P_{m}$. Some types of mutation operation includes Flip bit, Uniform and Gaussian mutations. In this work we adopt the uniform mutation, the value of the gene is changed between predefined upper and lower limits.

It is not necessary that good solutions will be created through crossover and mutation. However, only better solution will survive through the selection operator [9]. At the end of the evolution, the final non-dominated solutions are analyzed and used as feature extraction on unseen images.

\section{Experiments on Method I and II}

For robust image identification, triple features of an image should be very close to triple features of the distorted version of the same image. On the other hand, triple features of two different images should differ as much as possible.

In the experiment, a set of trace, diametric and circus functionals are used, which consists of 14 trace functionals $(T)$, six diametric functionals $(D)$ and six circus functionals $(C)$. Some of these functionals are listed in Table 1. Methods I and II are run individually to search for the best combinations of the Trace functionals for 200 generations. During the evolutionary stage, a set of five image classes are used with a low resolution of dimensions $64 \times 64$. Each class contains four images: original image and three distorted versions: rotated, scaled and translated (20 images in total). The original five images are displayed in the first row in Fig. 3. The population size and number of generations in method I and II are set to 150. There is no classifier training involved in this work. Table 2 depicts the parameters used in method I and II.

It should be mentioned that several independent runs of the two algorithms are performed and almost the same Pareto-fronts are achieved and the hypervolumes from different runs are almost the same. However, the final solutions may be reached at earlier generations but it continue as set to 200 generations. This conclude the randomness, yet, guided search of the evolutionary algorithms.

The experiments are performed on the same machine with Intel $\mathrm{R}$. Core ${ }^{\mathrm{TM}} 2 \mathrm{Duo}$ $3.1 \mathrm{GHz}$ processor with $3 \mathrm{~GB}$ RAM using Microsoft Visual $\mathrm{C}++$ compiler. The optimization time using method II took about 62 hours for 200 generations which is about a double the time in method I, which took about 29 hours. This is expected due to the double length of chromosomes in method II that requires running the Trace algorithm twice for each solution in the population. It is worth mentioning that this is an offline optimization of Trace transform aimed 
at finding out the good combinations of Trace transform functionals that might produce better triple features for image analysis in the online Trace algorithm which is itself takes a few seconds to process an image. The Pareto fronts at the final generation for both methods are depicted in Fig. 4. Undoubtedly, less solutions from method I are distributed at the convex area compared to method II which shows greater density in its Pareto front.

After 200 generations, there are nine solutions in total in method I. Each solution on the Pareto front of method I represents one triple feature to be combined with another solution to form a 2D feature space, whereas 19 solutions in the Pareto front of method II, each solution is equivalent to a pair of triple features which can form a 2D feature space directly. In method I, we construct up to 36 different pairs from the nine solutions to be evaluated using (4) and compared with solutions from method II. At this level, Fig. 5a shows the two equivalent fronts from method I (36 solutions) and method II. Obviously, both fronts are approximately identical, this can be concluded from the hypervolume indicator in Fig. 5b, where as little as $\simeq 0.11 \%$ hypervolume increase in method II is observed.

In the following, we investigate solutions on Pareto front of method I and II for the both objectives i.e. the within-class variance $S_{w}$ and the betweenclass variance $S_{b}^{-1}$. First, Fig. 6a depicts $S_{w}$ for solutions from both methods. A minimum (better) value can be seen from method II, whereas a maximum value can be identified from method I. Second, a maximum value of $S_{b}^{-1}$ can be identified from method II as shown in Fig. 6b. Generally, solutions have minimum value in one objective are not necessary have a minimum in the second objective. Additionally, one may also calculate the ratio of the two objectives as $S_{w} / S_{b}$ and are shown in Fig. 7. From the figure, a minimum value can be found in method I, whereas a greater maximum of this ratio can be found from solutions in method II. Keeping in mind, the 36 solutions in method I are thoroughly calculated by all possible combinations $\left(\begin{array}{l}9 \\ 2\end{array}\right)=\frac{9 !}{2(9-2) !}$ from the original nine solutions from the final Pareto front. This may be an easy task as few (nine) solutions were found in the final front of method I. However, it would have been a hard task if there were more solutions in the final front. Consequently, one may choose any two preferred solutions from the final front of method I to form a pair of triple features for image analysis, whereas solutions from method II can be used directly.

Table 2: Parameter Set-up for method I and II

\begin{tabular}{cc}
\hline \hline Parameter & Value \\
\hline Population size $N_{p}$ & 150 \\
Mutation probability & 0.125 \\
Crossover probability & 0.9 \\
Number of generations & 200 \\
$\epsilon$ & $10^{-5}$ \\
\hline
\end{tabular}


Next, we test the two methods on different images from fish database deformed by random rotation, scale and translation. Figure 3 depicts 20 original images (20 classes) used in the experiments. At this stage, each image has a standard dimension $256 \times 256$ and is subject to rotation, scaling and translation (distorted versions are omitted from the figure). Therefore, a total of 80 images are used (i.e. 20 classes, 4 images in each class).

Recall that the images in rows 2-4 of Fig. 3 (and their distorted versions) were not used in the evolutionary stage. Only the five images displayed in the first row of the figure (and their distorted versions) with a low resolution of dimensions $64 \times 64$ were used in the evolutionary stage of each method.

An example of features constructed from one solution picked up from each method I and II is shown in Fig. 8a and 8b respectively. We scaled these features to the interval $[0,1]$ by dividing features by a constant number. Assuredly, both figures show stable features and there is no overlap between any different classes. Moreover, each class shows compact features for different deformations of images belong to the same class.
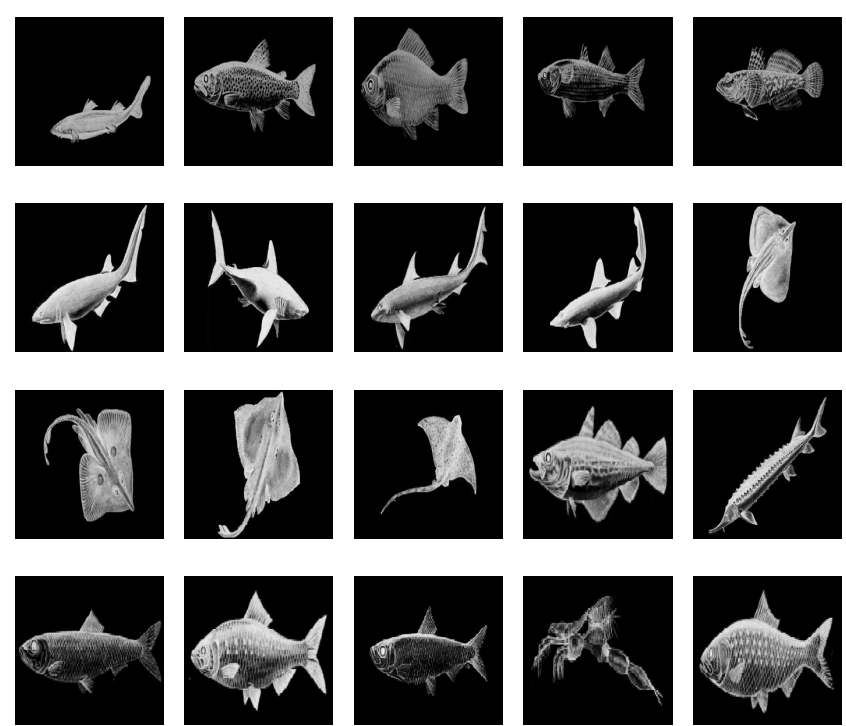

Fig. 3: Fish database [2]. Each image subjected to a random RTS deformation to form 80 image in total. 


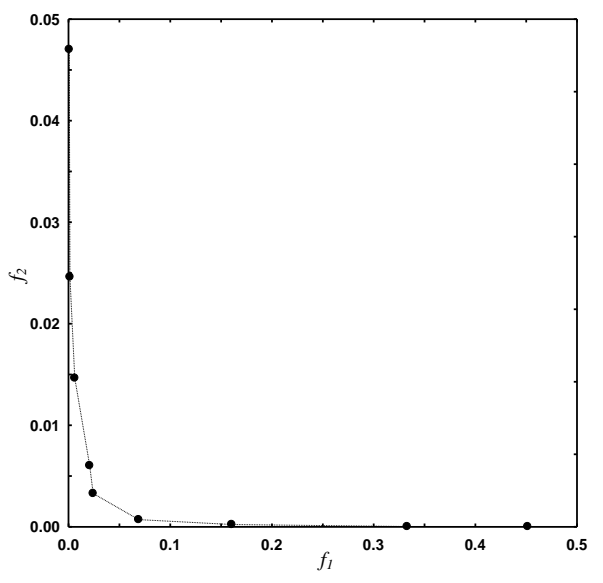

(a) Method I

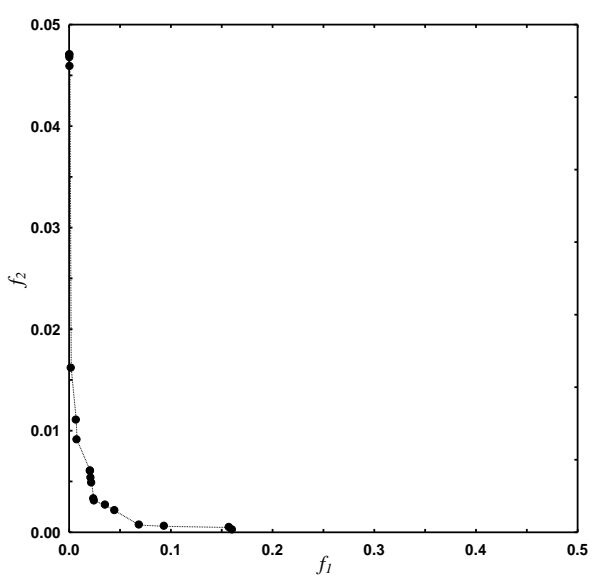

(b) Method II

Fig. 4: Non-dominated solutions as Pareto fronts in the objective space for method I and II after 200 generations.

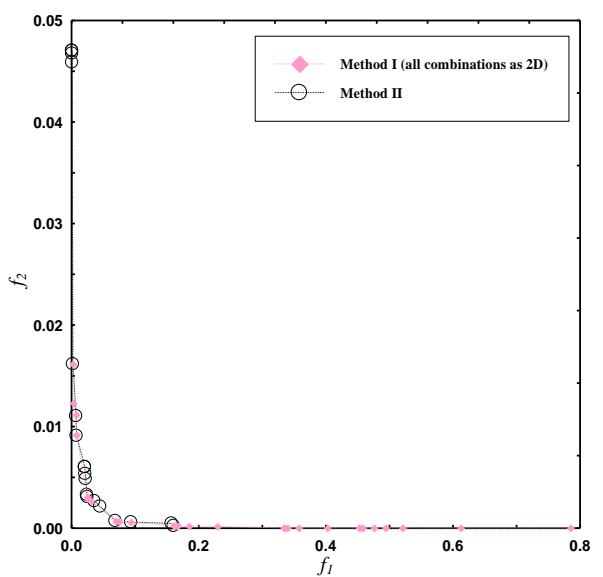

(a) Pareto fronts in the objective space.

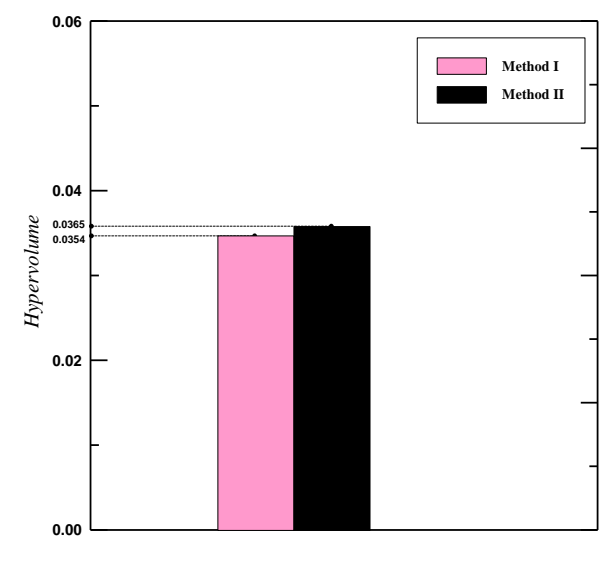

(b) Hypervolume indicator.

Fig. 5: Non-dominated solutions from method I(combined as 2D) and method II. 


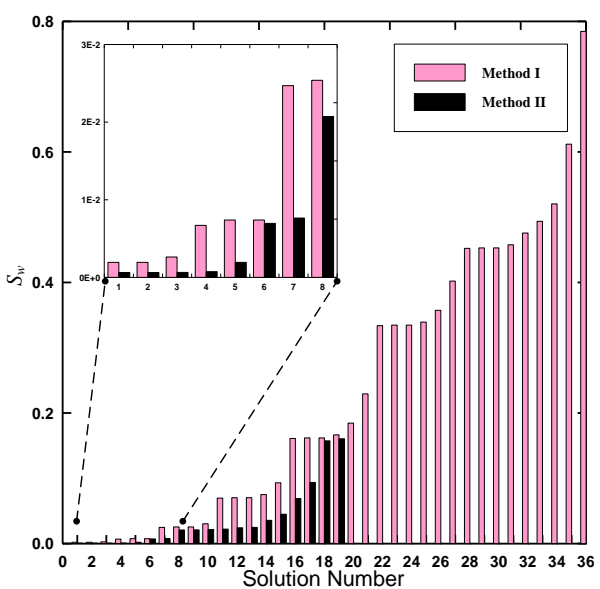

(a) Within-class variance $S_{w}$

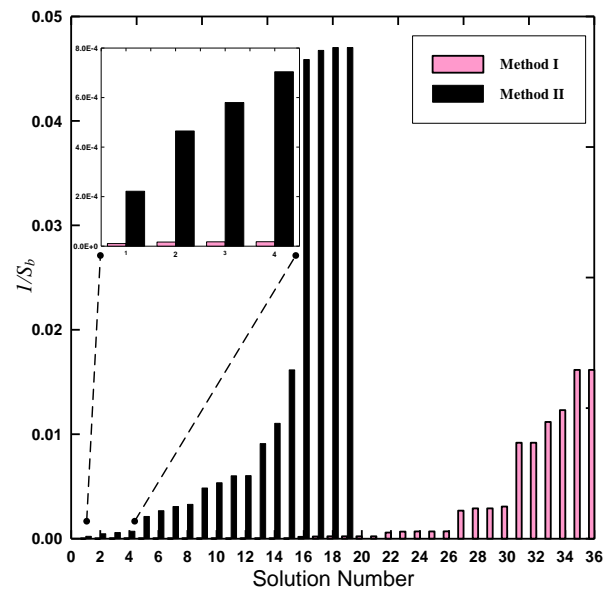

(b) Between-class variance $S_{b}^{-1}$

Fig. 6: The $S_{w}$ and $S_{b}^{-1}$ for solutions from method I and II.

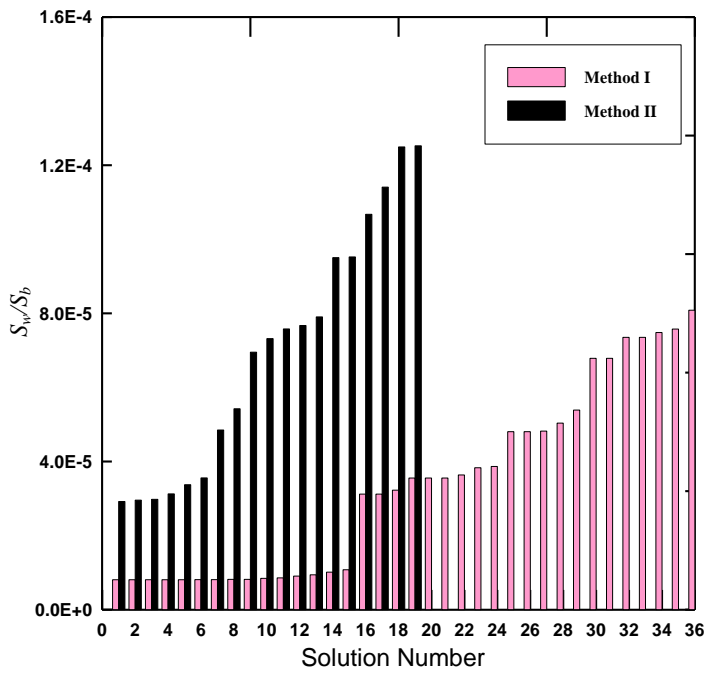

Fig. 7: The ratio of $S_{w} / S_{b}$ for method I and II. 
Wissam A. Albukhanajer et al.

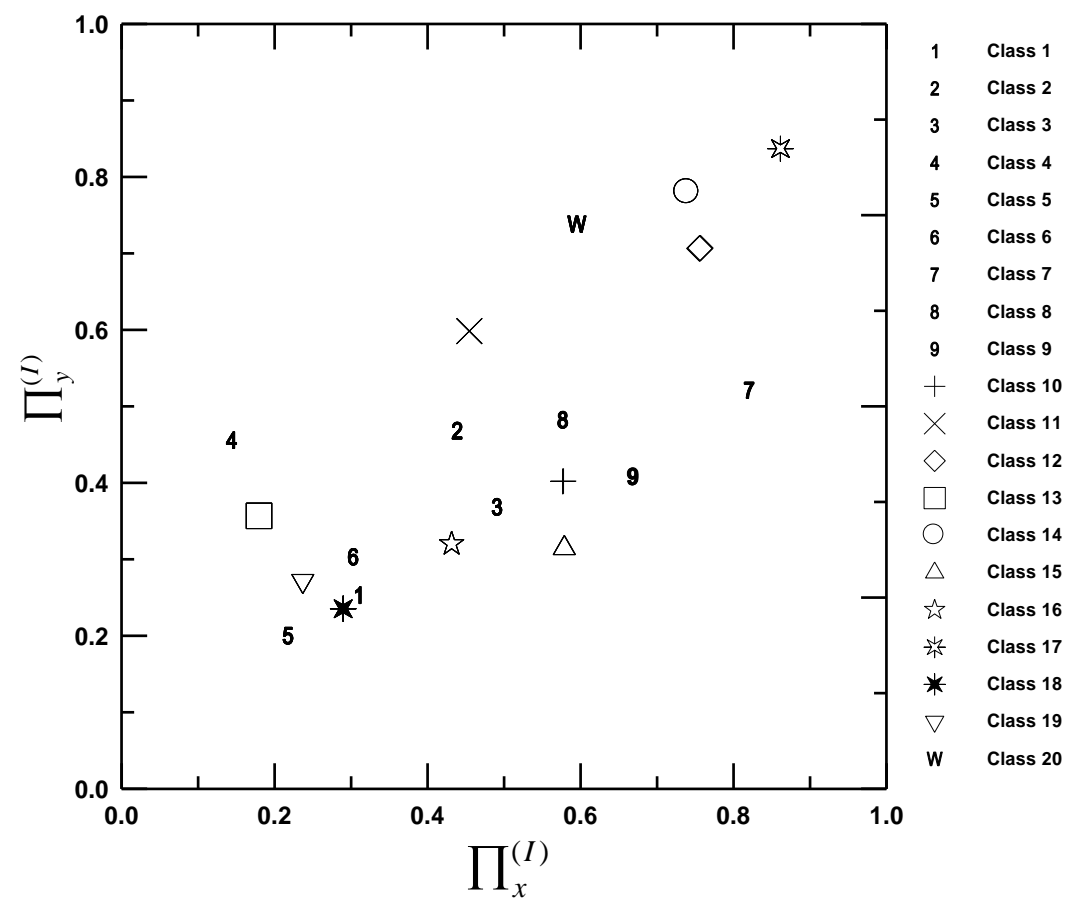

(a) Method I.

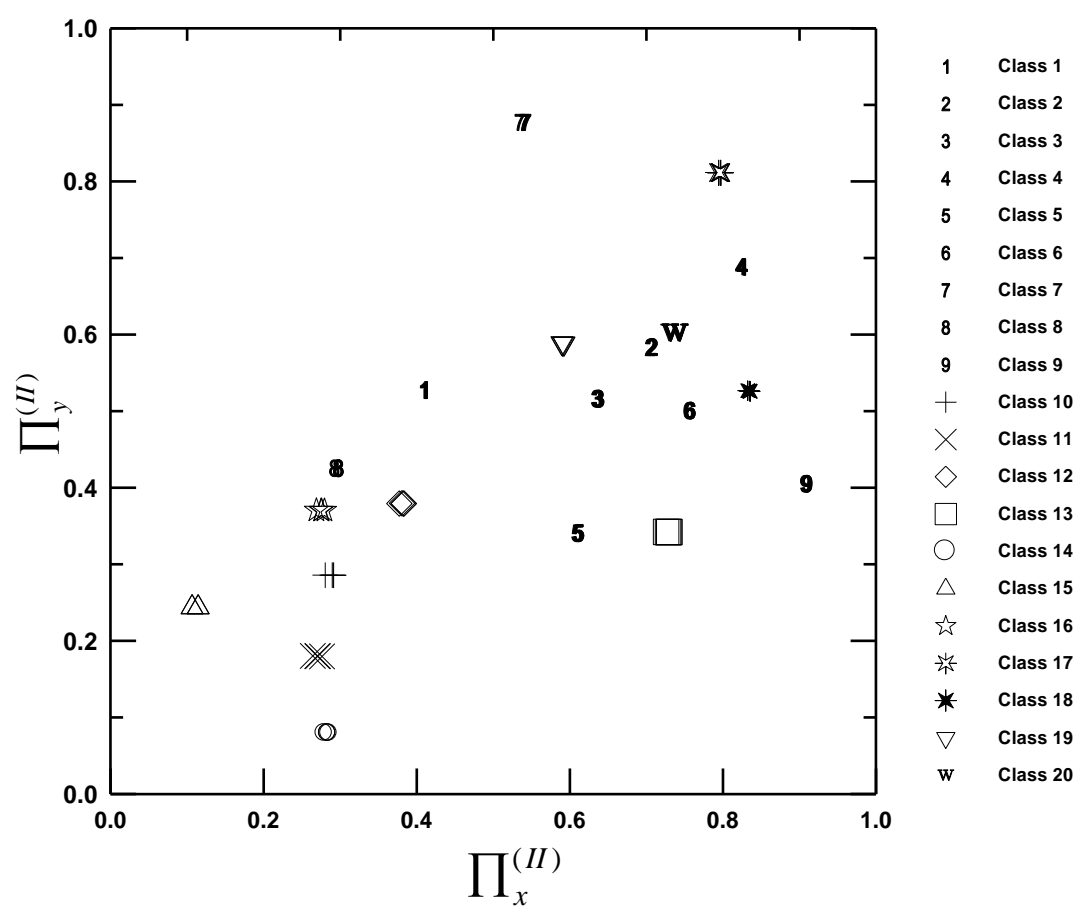

(b) Method II.

Fig. 8: Feature space using method I(combined as 2D) and method II. 


\section{Conclusion}

Two methods of evolving the Trace transform (method I and II) for robust image feature extraction are compared. In method I, one combination of functionals are optimized to extract 1D features. If 2D features are needed, two sets of functionals can be selected from the Pareto solutions to construct two 1D features. In method II, the Trace transform is optimized to directly extract $2 \mathrm{D}$ features. Therefore, each Pareto-optimal solution represents a pair of combinations of functionals, which can be applied to extract 2D features. A multi-objective evolutionary algorithm, NSGA-II, is employed to optimize the functionals in Trace transform. Method I and II uses a small number of low resolution $(64 \times 64)$ images for optimizing the functionals. Nevertheless, the optimized functionals have shown to work effectively to extract features from images of a high resolution. This indicates that functionals optimized offline using an evolutionary algorithm are able to extract features for robust identification of unseen images. The two methods are shown comparable results in terms of performance while method I is faster to evolve than method II. A set of 80 images form the fish database have been used to verify the effectiveness of method I and II. In the future, we plan to test the methods on various larger databases, and classifiers ensembles will be built based on the features extracted by ETT for image identification.

\section{Acknowledgements}

The authors would like to thank the anonymous reviewers for their insightful comments and suggestions that have significantly improved the quality of this paper.

\section{References}

1. B. Ruf, E. Kokiopoulou, and M. Detyniecki, "Mobile museum guide based on fast sift recognition," in Adaptive Multimedia Retrieval. Identifying, Summarizing, and Recommending Image and Music, ser. Lecture Notes in Computer Science, M. Detyniecki, U. Leiner, and A. Nurnberger, Eds. Springer Berlin / Heidelberg, 2010, vol. 5811, pp. 170-183. [Online]. Available: http://dx.doi.org/10.1007/978-3-642-14758-6_14

2. A. Kadyrov and M. Petrou, "The trace transform and its applications," IEEE Transactions on Pattern Analysis and Machine Intelligence, vol. 23, no. 8, pp. 811-828, 2001.

3. M. Petrou and A. Kadyrov, "Affine invariant features from the trace transform," IEEE Transactions on Pattern Analysis and Machine Intelligence, vol. 26, no. 1, pp. 30-44, 2004.

4. M. P. A. Kadyrov, A. Talepbour, "Texture Classification With Thousands of Features," in 13th British Machine Vision Conference. BMVC, 2-5 September 2002 2002, pp. 656-665.

5. M. F. Nasrudin, M. Petrou, and L. Kotoulas, "Jawi Character Recognition Using the Trace Transform," in Seventh International Conference on Computer Graphics, Imaging and Visualization (CGIV), 2010, pp. 151-156. 
6. W. A. Albukhanajer, Y. Jin, J. A. Briffa, and G. Williams, "Evolutionary MultiObjective Optimization of Trace Transform for Invariant Feature Extraction," in 2012 IEEE Congress on Evolutionary Computation (CEC), Brisbane, Australia, 10-15, June 2012, pp. 401-408.

7. N. Liu and H. Wang, "Extraction of hybrid trace features with evolutionary computation for face recognition," in IEEE Congress on Evolutionary Computation, (CEC 2007), 2007, pp. 2493-2500.

8. S. Srisuk, R. Fooprateepsiri, M. Petrou, S. Waraklang, and K. Sunat, "A general framework for image retrieval using reinforcement learning," in The Image and Vision Computing 2003, Nov. 26-28 2003, pp. 36-41.

9. K. Deb, Multi-Objective Optimization using Evolutionary Algorithms, 1st ed. England: John Wiley \& Sons. Ltd, 2002. 